\title{
The Ontological Interpretation of Quantum Mechanics
}

IN ANY MODERN EXPLORATION of the philosophy of science, one is at some point doomed to encounter that old, strangely constructed bridge that is quantum mechanics, the path across which seems to provide anything but sure footing. On one side of the chasm lie the conventional materialist ontologies, exemplified via the inductions of classical physics-inductions from our usual experiences of the macroscopic world. In general, these materialist ontologies characterize nature as fundamentally atomic and fluid-a collection of bits of matter whose motions, among other qualities and interactions, are deterministic and continuous through space and time. On the other side of the chasm lie modern experiences of the microscopic world, which, when initially accounted for by Planck and Einstein via the conventional materialist ontologies, characterized nature as a collection of material particles as before, but whose motions and interactions, when measured, appeared discontinuous and probabilistic rather than fluid and deterministic.

Bohr's 1913 model of the atom embodies this initial envisagement of the quantum mechanical microscopic world through the lens of mechanistic materialism. In this model, electrons are posited to be fundamentally material particles occupying a number of possible spatial "stationary states" around a fundamentally material nucleus. But rather than moving continuously through space from state to state according to previous conceptions, the electrons in Bohr's model must be thought of as making quantum leaps from one fixed state to another, each state associated with a discrete volume of space a certain distance from the nucleus and associated with a specific energy level. An electron making such a leap, in other words, must be thought of as making an instantaneous transition from one volume of space to another without moving through the space in between. Consideration of when or where an electron is during its 
transition from one state to another is rendered nonsensical in this model, despite the sensibility of such considerations given the materialist ontological framework Bohr's model otherwise requires. The demand for this troublesome caveat derives from the inescapable fact that any calculative prediction of an electron's state will yield only a probability as to which state it will occupy at the conclusion of a measurement, and never a unique factual outcome of the sort rendered by classical mechanics. And since the accuracy of these predicted probabilities is always confirmed retrodictively by experiment, the induction toward a "quantum" characterization of nature's most fundamental elements is, as concerns the science of physics, as justified as the materialist ontologies that preceded it.

The desire for a bridging of these two equally justifiable yet seemingly incompatible conceptions of nature - that of the conventional materialism of classical physics on one hand, and that inspired by the "old" quantum theory of Planck, Einstein, and Bohr on the other-fueled the subsequent work of Heisenberg, Bohr, Schrödinger, von Neumann, deBroglie, Dirac, Born, Jordan, and others during the years 1924-1928. But their efforts, while productive of a far more systematized and elegant quantum formalism, only served to emphasize and augment, rather than mitigate and reduce, the incompatibility of an ontological framework induced from classical mechanics and one induced from quantum mechanics. Granting for the moment that quantum mechanics is indeed ontologically significant at all, Abner Shimony distills these incompatibilities into five conceptual innovations implied by the quantum theory: (i) objective indefiniteness, (ii) objective chance, (iii) objective nonepistemic probability, (iv) objective entanglement, and (v) quantum nonlocality. ${ }^{1}$

Though the ontological implications of these innovations will be explored later in this chapter, it will be helpful to suggest now that there is a common source from which all five spring-a source that can be traced back even to the "old" quantum theory embodied by Bohr's atomic model: Though quantum mechanics makes use of facts - the facts constitutive of that which is being measured, for example, and the facts constitutive of the result of measurementquantum mechanics cannot account for these facts, nor are the "mechanics" of quantum theory productive of facts. The mechanics are productive of probabilities only. When used to predict and therefore 
describe a measurement interaction, quantum mechanics makes use of facts stipulated to exist antecedent to the measurement interaction-facts that account for that which is to be measured, as well as the apparatus that will perform the measurement. These facts are, according to the quantum formalism describing the measurement interaction, causally productive of a matrix of mutually exclusive and exhaustive probabilities - probabilities referent to a unique, factual outcome. Although this unique outcome is anticipated by the mechanics, it is not accounted for by the mechanics, and therein lies the key to the conflict among various competing interpretations of the quantum theory. For many theorists, typically those most heavily invested in the classical mechanical worldview, the failure of quantum mechanics to account deterministically for a unique outcome is indicative of the incompleteness of the theory. Thus the five conceptual innovations suggested by Shimony are, for such theorists, properly viewed as epistemological artifacts to be cleared up either by augmenting the theoretical formalism or by improving experimental technology.

For other theorists, the failure of quantum mechanics to account for the unique outcome states it presupposes and anticipates is really no failure at all; it is instead properly recognized as a logical, and indeed, ontological limitation operative in any scientific theory. With respect to a conceptually analogous case suggested by Murray Gell-Mann, ${ }^{2}$ when one wishes to predict the probability of a horse's winning a race, one necessarily presupposes (i) the fact of the horse (and everything else the race entails) antecedent to the race; and (ii) the fact of the horse's winning, or losing, at the conclusion of the race. By this view, even classical mechanics makes such presuppositions, which it cannot account for via its equations and formulasnamely, the very existence of the material particles whose motions and interactions are described by the mechanics.

That quantum mechanics is unable to account for its presupposed and anticipated facts has proven especially troublesome for some, given that quantum mechanics purports to characterize their relationship, via the Schrödinger equation, such that the matrix of probable measurement outcomes is not only subsequent to the measurement interaction and all the antecedent facts such an interaction presupposes, but also causally consequent of this interaction, which includes not only the facts constitutive of that which is mea- 
sured, but also the facts of the apparatus performing the measurement. John Bell states the difficulty thus:

When it is said that something is "measured" it is difficult not to think of the result as referring to some preexisting property of the object in question. This is to disregard Bohr's insistence that in quantum phenomena the apparatus as well as the system is essentially involved. If it were not so, how could we understand, for example, that "measurement" of a component of "angular momentum" ... in an arbitrarily chosen direction ... yields one of a discrete set of values?"

The outcome of a quantum mechanical measurement, in other words, is consequentially affected, at least to some degree, by that which measures-a conclusion described by Heisenberg's famous uncertainty relations. In their original 1927 incarnation, these relations were primarily intended to describe wave-particle duality with respect to position and momentum measurements and the Compton Effect manifest in such measurements: Measurement of the position or velocity of a particle, according to Heisenberg, necessarily entails the collision of the particle to be measured with particles associated with the measuring apparatus. These collisions will always affect position or velocity measurements such that, among other consequences, a simultaneous position and velocity measurement upon the same particle is impossible.

Given this particular application of the uncertainty relations, it might appear that they are more epistemically significant than ontologically significant - that they have more to do with a deficiency in the measuring procedure or apparatus than with the actualities being measured. However, a more generalized incarnation of Heisenberg's uncertainty relations in terms of the unavoidable change in a system's energy caused by a measurement interaction provides a more fundamental and ontologically significant description: For example, a measurement with duration $\Delta t$ is made of the energy of a system, and the measurement interaction causes an uncertainty or change $\Delta E$ in the energy measured, the magnitude of which is given by:

$$
\Delta E \Delta t \geq \frac{h}{4 \pi}
$$

where $h$ is Planck's constant.

Thus, the outcome of a quantum mechanical measurement is 
shown to be consequentially affected by that which measures. Unfortunately, however, this conclusion is often taken to imply that that which is measured via quantum mechanics is somehow causally influenced by that which measures - the source of the familiar lamentation that quantum mechanics has permitted the intrusion of sheer subjectivity into physics.

Such an alarming implication, however, should not be inferred from the Heisenberg uncertainty relations and the quantum formalism itself; it can only be inferred from the quantum formalism as interpreted via a classical materialist ontology, according to which a material object with factual material qualities antecedent to measurement continuously endures throughout measurement, thus remaining the same object at the conclusion of the measurement interaction-though by the Heisenberg uncertainty relations, with potentially different factual qualities somehow caused by the measurement. In other words, the object endures measurement by such an interpretation, yet its factual qualities change because of measurement and the particular apparatus used to perform the measurement. It is only when one attempts to interpret quantum mechanics via this classical materialist ontological notion of the qualification of an enduring substance by quality that quantum mechanics appears to thus threaten, via its supposed implication of sheer subjectivity, the objective reality of the world.

Alternatively, an ontological interpretation of quantum mechanics derived from the formalism itself, rather than from the formalism classically mediated, leads one toward a much different picture of the fundamental constituents of nature-again, granting for the moment that quantum mechanics is ontologically significant at all; one must keep in mind that all one can say for certain about quantum mechanics is that it is a theory by which physicists are able to successfully predict probabilities that specific types of measurements, under specific types of circumstances, will yield specific types of results. Whether or not this theoretical instrument is truly incapable of accommodation by the conventional materialist ontologies which otherwise reign supreme in physics is, to this day, a matter of heated debate, and arguments affirming a classical ontological interpretation of quantum theory have yet to be presented in this discussion. ${ }^{4}$ That stated, one can consider, even at this very early stage of the 
discussion, the following chain of reasoning productive of innovative ontological generalizations from the quantum formalism:

1. Quantum mechanics describes the evolution of initial facts antecedent to a measurement interaction to final novel facts subsequent to a measurement interaction. It describes this evolution via the linear, deterministic Schrödinger equation, which yields a matrix of probable novel facts, from which a unique final fact will obtain indeterministically. The term "measurement interaction," therefore, is a relational term that presupposes the objective existence of the facts thus related by the quantum mechanical evolution. Without these facts, there is nothing to relate, and the concept of measurement becomes meaningless.

2. Quantum mechanics reveals, per the Heisenberg uncertainty relations, that the facts subsequent to a measurement interaction are also consequent of the measurement interaction, and in this sense, facts presuppose measurement as much as measurement presupposes facts. Bohr thus rightly induces from quantum mechanics "the impossibility of any sharp separation between the behaviour of atomic objects and the interaction with the measuring instruments which serve to define the conditions under which the phenomena appear." According to quantum mechanics, then, the concepts of "fact" and "measurement" are mutually implicative; each is incapable of abstraction from the other, and both are therefore equally fundamental concepts. Put another way, facts are necessarily interrelated in quantum mechanics such that the notion of a single fact in isolation is meaningless.

3. Quantum mechanics therefore further reveals, per the Heisenberg uncertainty relations, that interrelations among facts are productive of novel facts, given that a measurement outcome is a novel fact (or an ensemble of novel facts) consequent of, and not merely subsequent to, the interrelations among facts comprising the measuring apparatus and the system measured. This is correctly interpreted not as a principle of sheer subjectivity, but as the fundamental physical exemplification of an ontological principle of relativity, such that any fact or system of facts cannot be considered apart from its interrelations with other facts. Measurement is an example of such interrelations.

Necessarily interrelated facts are therefore, according to the quantum formalism, the most fundamental constituents of nature capa- 
ble of description via the physical sciences-and hence, by ontological induction, the most fundamental constituents of nature herself. Apart from such an induction, quantum mechanical "facts" are merely epistemically significant in their role as purely subjective qualifications of a material substance by quality. According to any ontology strictly induced from the quantum formalism, then, all the materialistic conceptions of nature-conceptions, for example, of material bodies whose motions, among other qualifications, are continuous through space and time-become abstractions from the most fundamental constituents of nature, which are discontinuous, yet necessarily interrelated, quantum facts. The notion, then, of a classically "isolated" object of measurement in quantum mechanics cannot be taken as ontologically significant, but merely as a conceptual abstraction; a fact, by its ontologically necessary interrelations with other facts as induced from the quantum formalism, can never be isolated from other facts in the classical sense.

The obvious implication is that every measurement interaction somehow involves the entire universe of all facts-an idea clearly alluded to by Heisenberg, for example, when he writes, "the transition from the 'possible' to the 'actual' takes place as soon as the interaction of the object with the measuring device, and thereby with the rest of the world, has come into play."6 And indeed, a great many contemporary quantum theorists whose work will be explored in chapter 3 have demonstrated that the necessary interrelation among all facts in the universe is not only an interesting ontological induction one might draw from quantum mechanics; rather, theorists such as Robert Griffiths, Wojciech Zurek, Murray Gell-Mann, and Roland Omnès, among many others, have demonstrated that the interrelations between "measured" facts and facts belonging to the "environment" englobing the measurement interaction actually play a crucial mechanical role in the production of the measurement outcome.

\section{Bohr's Strategy: "Complementarity" as Evidence of an Unknowable Objective Reality}

The conception of necessarily interrelated facts as nature's most fundamental elements constitutes the first major departure from con- 
ventional materialist ontologies that hold that measurement necessarily presupposes fact- "measurement" in this sense being a qualification of a material substance by quality-but fact need not presuppose measurement, such that a fact, as well as the material body it qualifies, can be considered in isolation. Bohr's insistence that even quantum mechanical measurements must be made with a classically described apparatus exemplifies the initial attempt to at least partially mediate quantum mechanics with a classical materialist ontology in order to avoid this troublesome, mutually implicative relationship between fact and measurement. That this classical mediation prescribed by Bohr utterly contradicts his insistence on "the impossibility of any sharp separation" between the system measured and the apparatus measuring, as exemplified by the Heisenberg uncertainty relations, has of course been the source of a great deal of confusion; the difficulty manifests itself in the formalism, for example, when one attempts to account for the correlations between alternative potential microscopic measured-system outcomes and their respective alternative potential macroscopic measuring apparatus outcomes - a difficulty frequently referred to as "the problem of measurement" in quantum mechanics.

Indeed, most, if not all, of the conceptual difficulties and "paradoxes" associated with quantum mechanics can be traced to attempts to mediate or even wholly accommodate the latter within a classical mechanical ontology. There have been, over the years, a great number of proposed interpretations of quantum mechanics intended to solve these difficulties-some of which attempt to more coherently account for quantum mechanics as an abstraction from classical mechanics, and some of which attempt to account for classical mechanics as an abstraction from quantum mechanics. Many of these ontological proposals will be explored later in this chapter, but it will be useful here to introduce, and then quickly dispense with, an interpretation that stands apart from all others: the instrumentalist interpretation-more a mindset than an interpretation, given that it denies that any interpretation of quantum mechanics is necessary. It is a tradition advocated, in the words of Popper, by physicists who have turned away from interpretations of quantum mechanics "because they regard them, rightly, as philosophical, and because they believe, wrongly, that philosophical discussions are unimportant for 
physics.... [It is] a tradition which may easily lead to the end of science and its replacement by technology."

This tradition is sometimes confused with Bohr's "pragmatic interpretation" of quantum mechanics, which is unfortunate given Bohr's obvious interest in the philosophical implications of the quantum theory. Though to confuse the two would be unfair, it would certainly be fair to say that Bohr's pragmatic interpretation significantly inspired the instrumentalist mindset popular among many physicists today; for Bohr prescribes, as we have seen thus far, two utterly incompatible conceptions of a quantum mechanical measurement interaction, for no other reason than it is practical to do so. On the one hand, he explicitly proscribes the possibility of any "sharp separation between the behaviour of atomic objects and the interaction with the measuring instruments which serve to define the conditions under which the phenomena appear." other hand, Bohr prescribes treating the measuring apparatus classically, so that one is able to consider the facts pertaining to that which is measured as "external" to the apparatus, and therefore merely externally related, rather than mutually interrelated, thus preserving the conventional materialist notions of "subject" measuring "object." Likewise, the facts pertaining to the environment englobing that which is to be measured are similarly treated, such that the facts to be measured quantum mechanically can be considered as a "closed system," external to the surrounding environment.

An important justification for maintaining this classical conception of a closed, noninteracting system as the "object" of measurement is that the linear, deterministic Schrödinger equation at the very heart of quantum mechanics is applicable only to such closed systems. Bohr's prescription, however, also renders quantum mechanics nonuniversal and therefore ontologically insignificant, since the applicability of the mechanics becomes predicated upon an arbitrary dividing line separating the quantum and the classical.

That in itself may or may not be seen as a drawback, given that for practical purposes quantum mechanics is simply a tool used to predict the outcomes of measurements under specific conditionsnot universal conditions. However, given Bohr's insistence that the interrelations between the apparatus and the atomic objects measured render them inseparable, as expressed via the Heisenberg uncertainty relations, this utterly contradictory classical, subject-object 
ontological mediation of quantum mechanics necessarily implies the following: The object of measurement, though classically isolated from the subject which measures, is nevertheless influenced somehow by this subject. Again, it is this conclusion that has led many to lament that quantum mechanics severely vitiates, via its implied sheer subjectivity, the objective reality of the world-a grievous and paradoxical violation of the same materialist characterization of quantum mechanical measurement which predicates such a conclusion. The ontologically classical characterization of quantum mechanical measurement, prescribed by Bohr, implies a relationship between subject and object that violates this very characterization.

One may attempt to escape such a conclusion, however, by appealing to the previous conclusion that quantum mechanics is nonuniversal, and therefore ontologically insignificant-at least to the extent that it requires no ontological innovation; its significance is epistemological only. The Heisenberg uncertainty relations can therefore be interpreted as holding that only one's knowledge of that which is measured-knowledge yielded via the outcome of a measurement-depends on the apparatus performing the measurement. The apparatus does not influence that which it measures, but merely affects one's knowledge of that which is measured. That which is to be known about an object, then, depends on the questions asked. This pragmatic characterization of quantum mechanical measurement as prescribed by Bohr is a component of what has come to be commonly referred to as the Copenhagen Interpretation of the quantum formalism, and although it perhaps serves to mitigate the perceived intrusion of subjectivity into physics, it does not remove this intrusion altogether. For according to the ontological requirements of classical physics-which the Copenhagen Interpretation attempts to subsume at least conceptually in its intended role as "die endgültige Physik" (the "absolutely final" physics)—the knowledge of an object, gleaned via measurement, presupposes (i) the objective existence of the object measured; and (ii) that the knowledge of such an object constitutes a factual qualification of the object-that "a fact of knowledge" is "knowledge of a fact." Therefore, if the factual qualification of an object is affected by the subject making this qualification, as implied by quantum mechanics, and a factual qualification of an object derives from real "qualities" of the object as required by the conventional materialism of classical physics, then 
one must conclude, based on these premises, that qualifications of an object not only derive from the qualities of an object, but also affect the qualities of an object.

Therefore, even the characterization of quantum mechanics as merely epistemically significant does not prevent a violation of the classical, materialist ontology from which the premises of measurement-as prescribed by Bohr-derive. Even if one were to retreat to the notion that knowledge of an object gleaned via measurement does not necessarily reveal factual qualities pertaining to the object-that "a fact of knowledge" yielded by a quantum mechanical measurement does not necessarily entail "knowledge of a fact"- the objectively real material world thus immunized from sheer subjectivity is immunized only by the belief that the actual facts of such a world are essentially unknowable at all. Yet it is precisely this retreat which Bohr advocates as the best way to bridge quantum and classical mechanics: "In physics," he writes, "our problem consists in the co-ordination of our experience of the external world," such that "in our description of nature the purpose is not to disclose the real essence of phenomena but only to track down as far as possible relations between the multifold aspects of our experience."10

Thus, where the physical sciences were conventionally held to reveal factual qualities of nature, the objective reality of which was thought to be demonstrable by the apparently universal laws inducible from these qualities, Bohr suggests that quantum mechanics necessarily leads one to a much different conception of physics: The laws of physics, once thought to reveal the essence of nature's most fundamental constituents, now merely reveal subjective coordinations of our experiences of nature. "In quantum mechanics," Bohr writes, "we are not dealing with an arbitrary renunciation of a more detailed analysis of atomic phenomena, but with a recognition that such an analysis is in principle excluded" (emphasis in original). ${ }^{11}$ Physical laws, for Bohr, must be seen as qualifications of experience only, and the apparent regularity of those experiences capable of description by a physical law cannot be taken as evidence for or against a qualitative characterization of nature herself. For if one were to do so, one would be confronted with the fact that such inductions from the laws of quantum mechanics on one hand, and from the laws of classical mechanics on the other, each lead to entirely incompatible characterizations of nature. Better to say that the 
laws of quantum mechanics and classical mechanics reveal incompatible yet "complementary" - and for some unknown reason, exceedingly regular-coordinations of our experiences of nature. It is by means of such complementary coordinations of experience that one may bridge quantum and classical mechanics as mutually applicable to the task of characterizing our experiences of nature without characterizing nature herself.

Bohr's proscription against the induction of a fundamental characterization of nature from the laws of quantum mechanics-or classical mechanics-is the cornerstone of his proposed bridge linking quantum and classical mechanics; and in laying this cornerstone, he of course violates that very proscription. For it is clear that it was indeed quantum mechanics that led Bohr to impose the epistemic sanctions embodied by his "principle of complementarity" - and those sanctions clearly constitute an ontological characterization of nature whose essence, by this very ontology, consists of a fundamentally unknowable reality, ${ }^{12}$ subjectively experienced at two levels: (i) publicly, when these subjective experiences are regular enough to be described via physical laws which appear to hold universally; (ii) privately, when these subjective experiences are not regular enough to be described via a physical laws. These ontological generalizations, which Bohr clearly induced from quantum mechanics, and which clearly violate their own desiderata by the qualification of an "unknowable reality" as "unknowable," closely resemble, according to Henry Stapp, the ontology proposed by William James, ${ }^{13}$ which characterizes the world as consisting of (i) "hypersensible realities"; (ii) public "sense objects" (those subjective experiences whose coordination is regular enough to be expressible by physical laws); (iii) "private concepts" (those experiences that are truly "subjective" by the conventional meaning of the term, and incapable of coordination expressible by physical laws). ${ }^{14}$

The central claim of the Copenhagen Interpretation is that quantum mechanics is complete-that it cannot be abstracted from a more fundamental characterization of reality. Bohr's justification for this claim via the Jamesian-style ontology he prescribes, however, hinges on the even broader claim that reality is incapable of objective characterization at all. Since it is only one's experiences of reality that can be characterized and coordinated, the seeming incompatibility between the ontological implications and presuppositions of 
classical mechanics and those of quantum mechanics is resolved by stripping both of any ontological significance at all. Quantum and classical mechanics are thus relegated to the level of merely epistemically significant complementary coordinations of experience, and as such their incompatibility becomes unimportant. The practical advantages of this Jamesian ontological accommodation of the Copenhagen Interpretation are, then, as follows:

First, it allows for the classical conceptions of "subject" and "object" as externally related, such that a measurement outcome reveals the state of the object subsequent to the measurement interaction. One may, then, arbitrarily divide the components of a quantum mechanical measurement interaction into an object of measurement, classically isolated from the subject apparatus performing the measurement, as well as from the external environment englobing both. The evolution of the classically closed object/system through the measurement interaction is then capable of description via the linear, deterministic Schrödinger equation, which can be applied only to such a classically closed system.

Second, it allows for the nonclassical conceptions of subject and object as internally related-per the Heisenberg uncertainty relations - such that a measurement outcome reveals the state of both subject and object as consequent of, not merely subsequent to, the measurement. In this way, subject and object are nonclassically inseparable, since quantum mechanics describes the effect of the subject apparatus upon the measurement outcome, even when the apparatus and the object measured are spatially well separated.

These allowances were, for Bohr, thought to be possible only by virtue of his proviso requiring an ultimately unknowable reality, such that these wholly incompatible conceptions of subject and object in measurement do not constitute incompatible characterizations of the "real things" measuring and measured, which would render quantum mechanics incoherent and incomplete; they are instead to be considered only as complementary coordinations of our experiences of these things. Apart from this Jamesian ontological accommodation of the Copenhagen Interpretation, then, Bohr's "principle of complementarity" would amount to little more than an argument for the inescapable ontological incoherence of quantum mechanics, and therefore the completeness of quantum mechanics—the central 
claim of the Copenhagen Interpretation-would be anything but demonstrable.

\section{Von Neumann's Alternative to Bohr's Epistemic Schism: Objective Reality via a Coherent Ontological Interpretation}

The two advantages of the Jamesian ontology prescribed by Bohr are, however, readily achievable without adopting this conflicted ontology and its notion that reality is most fundamentally hypersensible and incapable of revelation via our experiences. And indeed, the completeness of the Copenhagen Interpretation-the notion that it entails the most fundamental characterization of our experiences of nature-is quite sensible apart from such an ontology. For as discussed at the beginning of this chapter, the most fundamental constituents of nature one might induce from quantum mechanics are necessarily interrelated facts from which all other conceptionsincluding classical notions of material objects with continuous motions and other attributes-can be shown to be abstractions. The ontological significance of the classical notion of an "objective reality" is preserved in the sense that interrelated facts are facts; measurement, as an example of such interrelation, presupposes the facts to be related-the fact of that which is measured, the fact of that which performs the measurement, and the anticipated fact of the outcome of the measurement. And the ontological significance of the Heisenberg uncertainty relations-wherein facts and measurement are mutually implicative-is guaranteed via the conceptually innovative notion of necessarily interrelated facts, such that the existence of a fact cannot be considered in isolation from other facts. In other words, since the fundamental constituents of nature are not just facts, but necessarily interrelated facts, fact presupposes measurement (interrelations with other facts) as much as measurement presupposes fact. The creation of a novel fact in quantum mechanics, in the form of a measurement outcome, requires and is conditioned by its interrelations with the facts antecedent to it-facts pertaining to that which is measured (system), but also to that which measures (apparatus) and, by implication, even to that which is not measured (environment).

According to such an ontological framework, an "experience of 
reality," as Bohr used the term, is ultimately the ontologically fundamental interrelation among facts-facts pertaining to we who experience, interrelated with the facts of the world we experience. The concept of "experience," then, is by this ontology identical to the concept of "measurement"; both are exemplifications of the ontologically fundamental notion of necessarily interrelated facts. An "experience of physical reality," given Bohr's use of the term, thus becomes a chain of interrelations: The interrelations of facts constituting that which is measured with the facts constituting the measuring apparatus; and the interrelation of facts constituting the apparatus with facts constituting the experimenter who observes the apparatus, et cetera. Bohr's concept of a hypersensible reality is no longer necessary, since experience itself-necessarily interrelated facts-is ontologically fundamental, rather than an underlying, essentially unknowable material "object" subjectively qualified by experience. The notion of quantum mechanical measurement as a chain of interrelated facts constituting that which is measured and that which measures, however, implies that the measuring apparatus-envisioned as a classical object by Bohr and thus in some sense isolated from the quantum mechanical system that it measuresshould instead be envisioned fundamentally as an ensemble of facts interrelated quantum mechanically with the facts being measured.

This was the approach proposed by von Neumann ${ }^{15}$ as a way of mathematically accounting for the correlations between the facts constituting the measuring apparatus and the facts constituting that which is measured. Moreover, if quantum mechanics is to be a truly coherent, universal theory, von Neumann suggests that these correlations should further extend to the facts constituting that which "measures" the measuring apparatus-in other words, the body and mind of the human observer. In this way, the classical conceptions of "subject of measurement" and "object of measurement" become properly understood as arbitrary abstractions from a more fundamental quantum mechanical characterization of measurement as the correlation of serially ordered quantum actualizations. Every particular subject-object correlation, then, becomes a datum for a subsequent subject-object correlation. It is only by such a scheme of "psycho-physical parallelism," suggests von Neumann, that the innovative and classically problematic "subjective" features of quantum 
mechanics might be mediated with the necessary "objective" realism in which modern science is grounded.

It is a fundamental requirement of the scientific viewpoint-the socalled principle of the psycho-physical parallelism-that it must be possible so to describe the extra-physical process of the subjective perception as if it were in reality in the physical world-i.e., to assign to its parts equivalent physical processes in the objective environment, in ordinary space. (Of course, in this correlating procedure there arises the frequent necessity of localizing some of these processes at points which lie within the portion of space occupied by our own bodies. But this does not alter the fact of their belonging to the "world about us," the objective environment referred to above.) In a simple example, these concepts might be applied about as follows: We wish to measure a temperature. If we want, we can pursue this process numerically until we have the temperature of the environment of the mercury container of the thermometer, and then say: this temperature is measured by the thermometer. But we can carry the calculation further, and from the properties of the mercury, which can be explained in kinetic and molecular terms, we can calculate its heating, expansion, and the resultant length of the mercury column, and then say: this length is seen by the observer. Going still further, and taking the light source into consideration, we could find out the reflection of the light quanta on the opaque mercury column, and the path of the remaining light quanta into the eye of the observer, their refraction in the eye lens, and the formation of an image on the retina, and then we would say: this image is registered by the retina of the observer. And were our physiological knowledge more precise than it is today, we could go still further, tracing the chemical reactions which produce the impression of this image on the retina, in the optic nerve tract and in the brain, and then in the end say: these chemical changes of his brain cells are perceived by the observer. But in any case, no matter how far we calculate-to the mercury vessel, to the scale of the thermometer, to the retina, or into the brain, at some time we must say: and this is perceived by the observer. That is, we must always divide the world into two parts, the one being the observed system, the other the observer. In the former, we can follow up all physical processes (in principle at least) arbitrarily precisely. In the latter, this is meaningless. The boundary between the two is arbitrary to a very large extent. In particular we saw in the four different possibilities in the example above, that the observer in this sense needs not to become identified with the body of the actual observer: In one instance in the above example, 
we included even the thermometer in it, while in another instance, even the eyes and optic nerve tract were not included. That this boundary can be pushed arbitrarily deeply into the interior of the body of the actual observer is the content of the principle of the psychophysical parallelism-but this does not change the fact that in each method of description the boundary must be put somewhere, if the method is not to proceed vacuously, i.e., if a comparison with experiment is to be possible. Indeed experience only makes statements of this type: an observer has made a certain (subjective) observation; and never any like this: a physical quantity has a certain value. ${ }^{16}$

One of the implications of this interpretation of quantum mechanical measurement is that the unique qualities of a particular actual "subject" in some way govern the correlations between that subject and its particular object. In this way, the set of probability outcomes yielded by quantum mechanics for any given measurement in a chain of measurements, such as the chain described above, "fits" the particular qualities of that subject within the chain. Von Neumann noted that the quantum mechanical mechanism governing these subject-object correlations is very different from the quantum mechanical mechanism by which a unique measurement outcome is actualized for any given measurement interaction. For one thing, the mechanism governing subject-object correlations does not yield a unique measurement outcome, but rather a mixture of probable measurement outcomes. This and other distinguishing features led von Neumann to further suggest that a coherent, universal interpretation of quantum mechanics requires that the process of subjectobject correlation in a quantum mechanical measurement interaction must be distinct from the process descriptive of a unitary wavefunction evolution in such an interaction. He thus proposes a "Process 1" productive of subject-object correlation and the influence of such correlation on the evolution of the mixture of probable measurement outcomes yielded by quantum mechanics; and "Process 2" describes the causal, unitary wavefunction evolution to a particular probable outcome state. Process 1, in other words, is a nonunitary and thus "non-causal" evolution explicative of the subjectobject correlation characteristic of the particular mixture of probable measurement outcomes yielded by this process; this particular mixture of probable outcome states, in other words, would have been otherwise if the subject had been otherwise. Process 2, by contrast, is descriptive of a more generic, unitary, and thus "causal" evolution 
of the wavefunction to a particular probable outcome state-an evolution von Neumann characterizes as an "automatic" change, as opposed to the "arbitrary" change effected by a specific subject-object measurement interaction described by Process 1 . Since quantum mechanics cannot account for the existence of actualities (though it can describe their evolution), Process 2 is merely descriptive of the unitary evolution from initial factual system state to final probable system state. But Process 1 is explicative, in that it accounts for the particular probability outcomes yielded; they are functions of the necessary quantum mechanical correlations between a particular subject (i.e., a particular measuring apparatus) and a particular measured system. Von Neumann writes:

Why then do we need the special Process 1 for the measurement? The reason is this: In the measurement we cannot observe the system $S$ by itself, but must rather investigate the system $S+M$, in order to obtain (numerically) its interaction with the measuring apparatus $M$. The theory of the measurement is a statement concerning $S+M$, and should describe how the state of $S$ is related to certain properties of the state of $M$ (namely, the positions of a certain pointer, since the observer reads these). Moreover, it is rather arbitrary whether or not one includes the observer in $M$, and replaces the relation between the $S$ state and the pointer positions in $M$ by the relations of this state and the chemical changes in the observer's eye or even in his brain (i.e., to that which he has "seen" or "perceived"). . . In any case, therefore, the application of [Process] 2 is of importance only for $S+$ $M$. Of course, we must show that this gives the same result for $S$ as the direct application of [Process] 1 on $S$. If this is successful, then we have achieved a unified way of looking at the physical world on a quantum mechanical basis. ${ }^{17}$

Von Neumann's "Process 1" and its relevance to the modern decoherence-based interpretations of quantum mechanics will be explored further in chapter 3. For now, let us return to his more general thesis that an ontologically coherent interpretation of quantum mechanics requires that both measuring apparatus and measured system be treated quantum mechanically. Though von Neumann's proposal would seem warranted by an ontology of fundamentally interrelated facts, the overwhelming complexity of a quantum mechanically described macroscopic measuring apparatus would entail calculations far too unwieldy, if not impossible, to be employed in practice. This criticism, however, cannot be taken as a sensible argument against 
his proposal; for the notion of necessarily interrelated facts as ontologically fundamental was induced from quantum mechanics by virtue of the fact that the formalism presupposes this notion. As mentioned earlier, quantum mechanics does not account for the existence of facts, nor can it, since a quantum mechanical description of a measurement interaction presupposes the existence of facts. By the same token, quantum mechanics does not explain the interrelation of facts; it presupposes this interrelation, and though it describes this interrelation, it cannot account for it. To fault quantum mechanics for its inability to explain the existence of necessarily interrelated facts presupposed by the mechanics is as unreasonable as to fault classical mechanics for its inability to explain the existence of matter, similarly presupposed. The notion of matter as ontologically fundamental was an induction made from classical mechanics and its description of matter; and the notion of necessarily interrelated facts as ontologically fundamental is an induction made from quantum mechanics and its description of these facts.

The conceptual advantage of von Neumann's approach is that it exemplifies the fundamental ontological concept that the process by which facts are interrelated should pertain to all facts-not just those facts which are arbitrarily "isolated," constituting that which is measured. The Schrödinger equation, which describes this interrelation, should apply to the facts comprised by the measuring apparatus as well as to the facts comprised by that which is measured. And, by implication, the facts comprised by the person observing (or "measuring" or "experiencing") the measuring apparatus should, by their interrelations with the facts of the apparatus and the system measured, also evolve according to the Schrödinger equation during the measurement interaction. Again, if the Schrödinger equation were used to describe the evolution of such a so-called von Neumann chain of interrelated facts, the calculations required would be entirely unmanageable; but conceptually, such a chain of interrelated facts is quite reasonable given the ontological inductions made thus far-and quite necessary if these inductions are to be coherent and consistent.

Von Neumann's program exemplifies the requirement, for the sake of ontological coherence, that the quantum mechanical interrelation of facts be universal, such that when we treat the measuring apparatus as a classical object as physicists do in practice, it is explicitly understood that the classicality of the apparatus is not an ontological 
characterization, but merely a conceptual abstraction from its more fundamental description as an ensemble of interrelated facts, which are themselves interrelated with the facts pertaining to that which is measured. So that when it seems as though the apparatus as a classical object, separated from that which it measures, somehow affects that which it measures, one is able to dispense with the abstraction and reclaim the underlying, ontologically fundamental notion of mutually and necessarily interrelated facts as the ultimate constituents of nature.

\section{Closed Systems}

We should recall, however, that treating the measuring apparatus as a classical object-whether as an abstraction from a more fundamental ontological conception, or as a "complementary" way of subjectively coordinating our experiences of a more fundamental "hypersensible reality" - is not merely to make the Schrödinger equation manageable; it is to make the Schrödinger equation applicable at all. For as mentioned earlier, the Schrödinger equation can be applied only to a classically "closed" system, and it is the treatment of the measuring apparatus as a classical object, isolated from that which it measures, which effectively renders the system measured "closed." It is, in this sense, difficult to suppose how an ontology based on mutually, necessarily interrelated facts can accommodate the requirement that some facts-those constituting the arbitrarily defined closed system-are incapable of interrelations with other facts. And since the Schrödinger equation lies at the heart of quantum mechanics, from which we have drawn our ontological inductions thus far, the coherence of these inductions depends upon their accommodation of this equation. For it is this equation that describes the mechanics of the interrelations among facts in a measurement interaction, and most significantly, it qualifies these relations as causally efficacious; without this qualification, then, quantum mechanics would be rendered utterly incapable of describing our experiences of physical causality.

The means by which a closed system can be abstracted from ontologically fundamental interrelated facts is quite simple, however, and is no threat at all to the coherence of this ontology; in fact, it is the requirement that the ontology be coherent and universal which provides the solution, already alluded to by von Neumann: If reality fundamentally consists of necessarily and mutually interrelated facts, 
then the only closed system capable of accommodation by such an ontology is, of course, the system of all facts. This would mean that the interrelations of some facts in a measurement interaction somehow entail the interrelations of all facts - that is, the entire universe. It would require, in other words, that even facts which are neither measured nor measuring-facts belonging to the "environment" englobing the measurement at hand-play some role in the quantum mechanical measurement of selected facts. Every quantum mechanical measurement, then, somehow must be thought to involve the entire universe-an implication, as indicated before, stressed by advocates of the decoherence-based interpretations of quantum mechanics that will be explored in chapter 3 .

But even von Neumann's proposal to treat the measuring apparatus quantum mechanically as an ensemble of interrelated facts was sufficient to render the calculations unmanageable. It therefore seems inconceivable that the experimental environment-let alone the entire universe-must be accounted for quantum mechanically as facts interrelated with the facts of the system measured and the measuring apparatus-particularly since the environment, unlike the measuring apparatus, seems to play no appreciable role in the orthodox quantum formalism. Although the universal interrelation of all facts is entirely justified as an implication of the ontological inductions made from quantum mechanics thus far, it is natural that those physicists who would embrace such inductions would want to see this implication exemplified functionally somehow by quantum mechanics itself. And indeed, as physicists, Griffiths, Omnès, GellMann, Zurek, and their like-minded colleagues stress the physical necessity of this implication-the quantum mechanical function of the interrelation between "environmental" facts and facts comprised by the system measured and the measuring apparatus-more than they do the philosophical necessity. And for them, environmental facts not only play a role in quantum mechanics, but a crucial role.

At this stage of the discussion, however, it will be helpful to emphasize that this notion of the universe as the only "closed system" involves an equally crucial conceptual innovation-one that is the hallmark of those interpretations of quantum mechanics which embrace this concept of "closed system": The "environment," via its necessary interrelations with all other facts, is thus able to "make measurements" as well as any apparatus or human observer, and in- 
deed does so continuously given the necessary interrelations of all facts within the closed system of the universe. Whereas "measurement" was typically characterized either tacitly or explicitly anthropically as the interrelations among facts comprised by a humanobserved "measuring apparatus" and facts comprised by a "measured system," the necessary interrelations of all facts within a closed system not only bring the facts comprised by the environment into play; the "measurement" of one "object" (subsystem of facts) by an "apparatus" (another subsystem of facts) in any given experimental procedure is now seen as a specific exemplification of the mutual interrelations among all facts, including those belonging to the environment, in the wider closed system.

This, of course, provides welcome relief to those who would lament that quantum mechanics entails the intrusion of necessary human subjectivity into physics; "facts as consequent of measurement" in quantum mechanics implied, for some, that facts would not exist at all unless measured by an observer-that the moon would exist with precise factual qualities such as position, size, shape, only when observed, or that a cat in a closed box containing a vial of poison that may or may not have spilled is somehow neither "factually alive" nor "factually dead" until the box is opened and the cat is observed. When the universe is properly considered as a closed system, however, one is able to acknowledge the interrelations between the cat and moon subsystems with facts environmental to these subsystems: Photons from the universal microwave background radiation are scattered off the moon, thus continuously "measuring" it; the molecules of the box interact with the cat as well as with the outside world, so the cat is similarly in a constant state of "measurement." Whereas a classical ontological conception of the cat, or any other item in the universe, characterizes it as an object which endures measurement (or the lack thereof), the ontological conception suggested by quantum mechanics characterizes the cat as a subsystem of facts in a state of perpetual creation by their interrelations with all other facts in the universe-a universe which, by the same process, is therefore itself in a perpetual state of creation. The classical conception of material existence thus becomes an abstraction from a more fundamental conception of creative factual interrelations.

In the closed system of the universe, all facts are interrelated, and 
therefore the interrelations of some facts-the measurement of one subsystem by another-necessarily entails interrelations with facts environmental to these subsystems. This "environmental monitoring" of measured systems in quantum mechanics is, as mentioned before, more than conceptually significant as regards the ontological coherence it affords to quantum mechanics; it is also directly and pragmatically reflected in the mathematical formalism of the mechanics, from which further interesting ontological inductions and hypothetical deductions such as those suggested in the paragraph above can be made, and that will be explored later in chapter 3 .

Leaving aside, for the moment, the formal quantum mechanical function of the interrelations between environmental facts and facts belonging to the system measured and the measuring apparatus, it is clear that consideration of the universe as the only truly closed system affords quantum mechanics a genuine ontological coherence and consistency only approximated or vaguely suggested by other interpretations. It is a coherence and a consistency far more substantial, for example, than that supposedly provided by Bohr's principle of complementarity, with its arbitrary dividing line demarking nature's quantum and classical essences as we experience and coordinate them via quantum and classical mechanics, respectively. And yet it must be emphasized that quantum mechanics does not merely entail the mutual interrelations among all facts comprised by the universe as the singular "closed system"; rather, quantum mechanics entails the interrelation of all facts antecedent to a given measurement interaction with facts subsequent to and, by the Heisenberg uncertainty relations, consequent of, this particular measurement interaction. Quantum mechanics, then, describes the interrelations among facts comprised by a closed system-the universe-such that these interrelations are always (i) productive of a consequential novel fact (the measurement outcome); and therefore (ii) always relative to that consequential novel fact, such that the novel fact in production is consequentially related with all facts antecedent to it.

\section{The Ontological Significance of Potentia}

Since, however, it is the production of a consequent novel fact-that is, a measurement outcome-that is conditioned by its interrelations 
with antecedent facts, and not the factual outcome itself (for as a "fact," a measurement outcome is already settled, "objectively real," and cannot be conditioned, influenced, altered, or undone), an ontology based on mutually related facts requires an additional innovation-the concept of a potential fact. Potential facts as mathematical components of the quantum formalism provide the means by which the creation of a novel fact, such as a measurement outcome, can be causally related with antecedent facts in quantum mechanics.

Heisenberg, in his analysis of the ontological significance of quantum mechanics, insists upon the fundamental reality and function of potentia in this regard. For him, potentia are not merely epistemic, statistical approximations of an underlying veiled reality of predetermined facts; potentia are, rather, ontologically fundamental constituents of nature. They are things "standing in the middle between the idea of an event and the actual event, a strange kind of physical reality just in the middle between possibility and reality."18 Elsewhere, Heisenberg writes that the correct interpretation of quantum mechanics requires that one consider the concept of "probability as a new kind of 'objective' physical reality. This probability concept is closely related to the concept of natural philosophy of the ancients such as Aristotle; it is, to a certain extent, a transformation of the old 'potentia' concept from a qualitative to a quantitative idea." 19

Quantum mechanics thus becomes characterized as the mechanics of interrelations among facts - among "actualities" - toward the production of novel actualities (i.e., measurement outcomes), and these interrelations are mediated by potentia-the real "things" upon which the mechanics operate. Therefore, a coherent ontology induced from quantum mechanics as suggested by Heisenberg in the preceding quotations presupposes not one but two fundamental constituents of nature-two species of reality: (i) necessarily interrelated facts, or "actualities"; (ii) potential facts, or "potentia," which provide the means by which a novel fact is causally interrelated with the facts antecedent to it. One would expect that if potentia are, indeed, one of the two fundamental species of reality implied by quantum mechanics, that potentia and their ontological function would find some exemplification in the quantum formalism. And they do, in the form of the matrix of probabilities yielded in a quantum mechanical measurement interaction. These probabilities, terminal of every quantum mechanical measurement interaction, are 
understood to be potential outcomes-potential facts which will be constitutive of the measured system after measurement.

When one speaks of the "state" of a system, one is referring to a maximal specification of these facts, so that in quantum mechanics one is concerned with (i) the initial, actual state of the system prior to the measurement interaction; (ii) the matrix of mutually exclusive and exhaustive potential outcome states predicted by quantum mechanics, each of whose propensity for actualization is valuated as a probability; and (iii) the actual, unique outcome state observed at the end of the measurement. It is always the case that only a single outcome state is ever observed-that only one potential outcome state ever becomes actual in any given measurement interaction. But it is also the case that the "actualization" of this unique potential outcome lies beyond the scope of quantum mechanics, which yields only a matrix of probable outcomes and never a singular, determined outcome. This difficulty is typically referred to as the "problem of state reduction" or the "problem of the actualization of potentialities."

One might connote in these two terms the idea of a physical, dynamical reduction mechanism which should be thought to operate upon the matrices of potentia yielded by a quantum mechanical measurement interaction. For if potentia are indeed ontologically significant-that is, "real"- there should be, it has been suggested, some equally "real" physical mechanism describing their dynamical evolution to a unique, actual state, in the same way that the Schrödinger equation describes the dynamical evolution from the initial, unique, actual state to the matrix of potential outcome states. Quantum mechanics, by such an argument, is therefore incomplete and in need of augmentation. Some physicists have developed a variety of proposals to this end-stochastic and nonlinear modifications of the Schrödinger equation intended to account mechanically for the actualization of potentia. The ontological implications of some of these programs will be explored presently, but for now it is enough to note that these proposed remedies for the incompleteness of quantum mechanics were inspired by the belief in the ontological significance of potentia as suggested by Heisenberg. The difficulty is that these proposals treat potentia as though they were actualities, conflating the two concepts such that the Schrödinger equation is interpreted as producing "coexistent" actual alternative measure- 
ment outcomes that must, then, be physically and dynamically reduced to a single outcome.

The conceptual difficulties produced by such conflation of actuality and potentiality in quantum mechanics were most notably forewarned by Schrödinger himself in his infamous "cat" hypothetical, conveyed in a brief paragraph of his 1935 essay "Die gegenwartige Situation in der Quantenmechanik." ${ }^{20}$ A cat, placed in a box, is subjected to a procedure wherein it will either live or die as a result. A quantum mechanical measurement of the cat, then, yields a matrix of two probability outcomes. The problem of state reduction manifests here in two questions: (i) If quantum mechanics is ontologically significant, then the matrix of two probability outcomes yielded (catalive and cat-dead) must be ontologically significant; if this is so, then why do we never experience the monstrosity of a live-dead cat superposition terminal of a quantum mechanical measurement? (ii) Since a quantum mechanical measurement of the cat yields such a matrix or superposition of coexisting states, yet observation always yields a unique state wherein the cat is always either dead or alive and never both, what is the physical mechanism by which the superposition or matrix of coexistent, "real" states is reduced to a unique "real" state, and when is this mechanism effected? Many physicists have concluded that, as mentioned earlier, the Schrödinger equation requires modification to account for this elusive mechanism; for unless one were inclined to assign the same ontological significance to "potentia" as is assigned to "actualities" in classical mechanics, one must interpret the matrix of probable outcome states yielded by quantum mechanics as a matrix of coexisting actual states.

The conception of potentia as a separate species of realityontologically, rather than merely epistemologically significant-has not been widely embraced by physicists biased toward a classical ontological accommodation of quantum mechanics. And indeed, most of the proposed solutions to the problem of state reduction entail attempts to fit quantum mechanics into a classical ontological framework. Many of these attempts have proven to be extremely unappealing because they either entail presuppositions even more radical than the concept of "real" potentia suggested by Heisenberg and championed by Popper, or because they have been experimentally disconfirmed. They are, nevertheless, important to any exploration of the ontological significance of quantum mechanics, for they 
have helped to disclose the standards of coherence, logical consistency, empirical applicability, and empirical adequacy sought after in its interpretation.

The particular classical, materialist ontological interpretations of quantum mechanics referred to above characterize the matrix of probable measurement outcomes as a set of coexistent, actualized (classically real) alternatives, the superposition of which is dynamically reduced by some physical process to yield a unique actuality. The proposal of Eugene Wigner is representative of this approach to state reduction. For Wigner, the continuous evolution of the state of the measured system as described by the Schrödinger equation is inconsistent with the seemingly discontinuous actualization of a unique outcome state. He suggested that this inconsistency might be remedied by a nonlinear modification of the Schrödinger equation, which would account for a dynamical mechanism by which the matrix of alternative real states is discontinuously reduced to a single state. Wigner further suggested that the mechanism described by this nonlinear modification might even be attributed to the influence of the mind of the observer upon that which is measured, which would account for why we never observe superpositions or matrices of alternative states in nature; for the act of observation itself is the nonlinear mechanism causative of state reduction. ${ }^{21}$ One finds a similar conclusion given by Walter Heitler, who wrote: "One may ask if it is sufficient to carry out a measurement by a self-registering apparatus or whether the presence of an observer is required." Heitler concluded that "the observer appears, as a necessary part of the whole structure, and in his full capacity as a conscious being." 22

It should be stressed that despite occasional assertions to the contrary, Heisenberg did not ultimately hold this view as an appropriate induction from quantum mechanics. For Heisenberg, again, potentia are ontologically significant constituents of nature that provide the means by which the facts comprising the measuring apparatus and the facts comprising the system measured (as well as those comprising the environment) are interrelated in quantum mechanics: "The transition from the "possible' to the 'actual' takes place," he writes, "as soon as the interaction of the object with the measuring device, and thereby with the rest of the world, has come into play; it is not connected with the act of registration of the result by the mind of the observer."23 The conflation of Heisenberg's ontological inductions and those of Wigner, Heitler, and others is, however, under- 
standable; for Heisenberg's ontologically innovative contribution to the Copenhagen Interpretation was amalgamated with the severe epistemic sanctioning of Bohr's worldview. Heisenberg thus interprets the matrix of probable outcome states not as an objective integration of potentia-despite his belief that potentia are "real" in the Aristotelian sense-but rather as a mathematical representation of our incomplete knowledge of the evolution of the measured system. Though he clearly holds that, ontologically, the integration of potentia is productive of, and will "reduce to," a unique actuality, the density matrix itself is, for Heisenberg, merely an epistemic reflection of these potentia. And the discontinuous reduction of the density matrix is, likewise, an epistemic reflection of an increase in one's knowledge of the facts that have actualized. Heisenberg explains that although the ontological actualization of potentia is not driven by any physical mechanism associated with the conscious registration of the actualized by the mind of the observer, "the discontinuous change in the probability function, however, takes place with the act of registration, because it is the discontinuous change of our knowledge in the instant of registration that has its image in the discontinuous change of the probability function." 24 Heisenberg's concept of potentia thus seems to imply both an unavoidable subjectivity veiling nature and, at the same time, her reliable objective reality. Patrick Heelan explains this dual function thus:

The objective tendency or potentia ... is on the one hand not simply the thing-in-itself in the external world, nor on the other hand is it simply the transcendental ego; it bridges both the external world and the transcendental subjectivity of the knower. As Heisenberg wrote in the Martin Heidegger Festschrift (1959), "the search for the natural laws of the [ultimate structure of matter,] entails the use of general principles of which it is not clear whether they apply to the empirical behaviour of the world or to a priori forms of our thought, or to the way in which we speak." 25

Most of the confusion with respect to Heisenberg's concept of potentia and its dual implication of subjectivity and objectivity in nature has to do with the habitual classical tendency to apply the fundamental mediative function of potentia solely to facts belonging to (i) "measured system" and (ii) "measuring apparatus"- that is, the classical "object" and "subject"; the result is that the broader, 
more accurate role of potentia is overlooked. For a coherent ontological interpretation of quantum mechanics reveals that most fundamentally, potentia are mediative of facts, whether they belong to the measured system, the measuring apparatus, the human observer, or the universal environment-for all are subsumed by the singular closed system of the universe. It is not the subjective interaction of "human" facts with object "measured system" facts that somehow alters or actualizes the latter; it is, rather, the interrelations among all facts relative to a given fact or subsystem of facts, which are productive of potentia which will evolve to become novel facts. With their most fundamental role in mind, then, it is clear that Heisenberg's potentia are not indicative of a fundamental subjectivity pervading nature, but rather a fundamental relativity.

For many, Heisenberg's epistemological interpretation of the density matrix has overshadowed and obfuscated the ontological implications he proposed - the reality of potentia and the idea that novel actualities are somehow produced by a process involving the integration and valuation of these potentia. The conflation of his interpretation of state reduction and those of Wigner, Heitler, and others, is an unfortunate consequence of this obfuscation, but understandable in light of it. Much theoretical work has been done in recent years, however-proposals by several physicists mentioned earlier, such as Roland Omnès, Robert Griffiths, Murray Gell-Mann, and Wojciech Zurek, to name a few-which begins with Heisenberg's ontological interpretation of potentia. These theories then depict, explicitly, the evolution of the state of a measured system-the integration and valuation of the potentia associated with this system-as an ontological process, rather than merely an epistemological representation of an underlying ontology, as given by Heisenberg. These interpretations will be explored more fully in the following chapter, but they are mentioned here to suggest that the ontological characterization of state reduction implied by Wigner and Heitler, wherein the density matrix is depicted as an integration of coexisting actualities reduced by some physical mechanism to a unique actuality, is not the only ontological characterization that physicists have considered. For the depiction of the density matrix as an integration of coexisting actualities, though it would certainly render quantum mechanics ontologically significant, exacts a price many physicists are unwilling to pay-the implication that until the box containing Schrödinger's cat 
is opened, the cat exists as a superposition of two alternative, yet equally actual states.

Yet for many physicists, this price is entirely reasonable given the undeniable value of an ontological interpretation of quantum mechanics. The "Continuous Spontaneous Localization" theory of GianCarlo Ghirardi, Alberto Rimini, and Tulio Weber (GRW), based on early work by Phillip Pearle in the 1970s, also treats the superposition of states yielded by the Schrödinger equation as a superposition of actual physical states, upon which there should operate some physical mechanism causative of reduction to a unique state such that macroscopic superpositions like Schrödinger's cat are extremely short-lived. Like Wigner, GRW propose a modification of the Schrödinger equation to this end; but whereas Wigner proposed a characterization of state reduction as ultimately discontinuous via a nonlinear modification, the spontaneous localization theory characterizes state reduction as ultimately continuous, via a linear modification. The proposed mechanism for unitary reduction represented by this modification is a stochastically fluctuating field, which continuously and spontaneously "collapses" the superposition of states into a unique state. ${ }^{26}$ Where particle density is high, as in the case of a macroscopic object such as a cat, these stochastic field fluctuations cause extremely rapid collapses such that, according to John Bell, "any embarrassing macroscopic ambiguity in the usual theory is only momentary in the GRW theory. The cat is not both dead and alive for more than a split second." 27 However, the disconcerting ontological implications of the cat's being simultaneously alive and dead at all, even if for only a split second, cannot be overlooked. Among other difficulties is the implied double-violation of the logical principles of non-contradiction and the excluded middleviolations which undermine one of the first principles of modern science: the presupposed correlation of causal relation and logical implication.

And indeed, the spontaneous localization theory of GRW is intended to be ontologically significant given that it purports to specify "the physical reality of what exists out there"28 according to Ghirardi. The spontaneous localization theory is to provide, he writes, "a mathematically precise formalism allowing a unified description of all phenomena, containing a single fundamental dynamical principle that governs all processes." 29 Thus, the theory not only implies the coexistence of alternative versions of entities like Schrödinger's cat, 
even if only for a split second; even more troubling, it entails an ontology whose "fundamental dynamical principle" is the continuous destruction of most of these entities. Indeed, it would seem that any interpretation of quantum mechanics that treats the density matrix as comprising, against Heisenberg's admonition, coexisting, alternative actualities rather than coexisting potentia, would require an ontological principle whereby the vast majority of these entities are destroyed-whether continuously, per GRW's proposal, or discontinuously, per Wigner's proposal.

The "Relative State" interpretation of Hugh Everett-often referred to as the "many worlds interpretation" 30 — purports to avoid this troubling ontological implication. It is similar in its treatment of the density matrix as an amalgam of coexisting, actual alternative states; but whereas the interpretations discussed earlier proposed the need for a physical mechanism to reduce this matrix to a unique state, Everett suggests that no such mechanism or modification of the Schrödinger equation is necessary, given that the concept of a single unique outcome state is itself unnecessary. We never experience a superposition of equally real alternative states because each alternative state can be thought of as a unique outcome state occurring in its own relative universe. An alternative state is, in this way, always relative to its particular universe in the same way that a temporal duration, by the theory of special relativity, is always relative to its particular inertial frame.

A key advantage of Everett's proposal is that it provides a conceptual account of the correlations between the facts comprised by the measuring apparatus and those comprised by the system measured; like von Neumann, Everett suggests that both the apparatus and the system measured evolve quantum mechanically. But whereas von Neumann was unable to account for the correlations between the separate evolutions of the system and apparatus, Everett is able to do so by virtue of the fact that each probable system outcome, and its correlated apparatus outcome, occurs in the same "relative universe." One universe contains a live cat, correlated with an observer who sees a live cat, and another universe contains a dead cat, correlated with an observer who sees a dead cat.

Though Everett's proposal avoids the implicit ontological principle by which most coexistent, alternative entities must be destroyed, there remains the difficulty of accepting an ontology that entails that there be multiple "copies" of oneself_an ontology where there is no 
distinction between potentiality and actuality. Such an implication is arguably far more radical and philosophically problematic than that suggested by Heisenberg, wherein potentia are fundamentally "real," though not "actual," constituents of nature. Indeed, an ontology where everything that can happen does happen renders meaningless the idea of things that should happen. "If such a theory were taken seriously," writes John Bell, "it would hardly be possible to take anything else seriously. So much for the social implications." 31

Common to all these proposals and a great many others is the conflation of actuality and potentiality symptomatic of any attempt at a classical accommodation of quantum mechanics. The "reality" of the alternative probability outcomes yielded by quantum mechanics is indeed acknowledged in these theories, but it is reality as classically defined-reality monopolized by actuality-such that Schrödinger's cat in its bizarre live-dead superposition is necessarily as real as any other cat. Heisenberg's conception of potentia as ontologically significant, fundamental constituents of nature answers this mischaracterization, such that the matrix of alternative states yielded by quantum mechanics is a matrix of coexisting potentialbut nevertheless real-states, not a matrix of coexisting actual states. For Heisenberg, the concept of potentia, then, constitutes, in his words,

a first definition concerning the ontology of quantum theory. ... One sees at once that this use of the word "state," especially the term "coexistent state," is so different from the usual materialistic ontology that one may doubt whether one is using a convenient terminology. ... One may even simply replace the term "state" by the term "potentiality" - then the concept of "coexistent potentialities" is quite plausible, since one potentiality may involve or overlap other potentialities. $^{32}$

The problem of state reduction thus becomes redefined: The question is no longer, "What is the mechanism by which a unique actuality physically evolves from a matrix of coexistent actualities?" but rather, "What is the mechanism by which a unique actuality evolves from a matrix of coexistent potentia?"

The answer to that question can be found in the quantum formalism itself: The matrix of states yielded by quantum mechanics is not merely a matrix of potential states; they are, rather, mutually exclusive and exhaustive probable states. That is to say, they are a selection 
of potential states that have evolved, via the Schrödinger equation, to become probabilities - that is, potentia qualified by a valuation between 0 and 1 , such that together, these probable states represent mutually exclusive and exhaustive potential outcomes, satisfying the logical principles of non-contradiction and the excluded middle, respectively. Thus one (and only one) outcome must occur. Unlike purely potential outcomes, then, probability outcomes clearly presuppose and anticipate the necessary actualization of a unique outcome; for without such an outcome, the concept of a probability itself is utterly meaningless. In this sense, the actualization of potentia is less a physical, dynamical function of quantum mechanics than it is a conceptual function logically presupposed by the mechanics.

As stated earlier, to attempt to account for the existence of facts via a mechanism that presupposes their existence is logically untenable. Nevertheless, the inability of quantum mechanics to account for a unitary evolution from a pure-state superposition to a unique measurement outcome-rather than to a matrix of alternative probable outcomes - is considered by many physicists to be a defect of the formalism, or as we have seen in the examples above, at least a problem that should be solved. Against such objections, we may simply recall the previously cited analogy suggested by Murray GellMann: When one wishes to predict the probability of a horse winning a race, one necessarily presupposes (i) the fact of the horse (and everything else the race entails) antecedent to the race; and (ii) the fact of the horse's winning, or losing, at the conclusion of the race. In the same way, quantum mechanics cannot account for the existence of facts, given that (i) quantum mechanics presupposes their existence antecedent to the measurement interaction-facts which account for that which is to be measured, as well as the apparatus which will perform the measurement; and (ii) quantum mechanics anticipates their existence, via the yielded mutually exclusive and exhaustive probability outcomes, subsequent to and consequent of the measurement interaction.

Put simply, quantum mechanics does not describe the actualization of potentia; it only describes the valuation of potentia. The answer to the problem of state reduction is thus at once as simple and as elusive as the "problem" of the three interior angles of a triangle adding up to 180 degrees, or the "problem" of the existence of the universe in classical mechanics. In the case of the latter, most cos- 
mologists have found it much more interesting to simply logically stipulate the existence of the primordial $t=0$ initial conditions of the universe, in whatever form it may have had, and focus instead on the mechanics describing how those initial conditions have evolved to become what the universe is today, and thus glean what it might become tomorrow. Similarly, in quantum mechanics, the "problem of the actualization of potentia," or more generally, the problem of the existence of facts, is far less interesting than the mechanics that describe how facts are causally productive of potentia, and how these potentia evolve to become valuated probabilities subsequently and consequently anticipative-and somehow creative-of novel facts.

\section{Notes}

1. A. Shimony, "Search for a Worldview Which Can Accommodate Our Knowledge of Microphysics," in Philosophical Consequences of Quantum Theory: Reflections on Bell's Theorem, ed. J. Cushing and E. McMullin (Notre Dame, Ind.: Notre Dame University Press, 1989), 27.

2. Murray Gell-Mann, The Quark and the Jaguar: Adventures in the Simple and the Complex (New York: W. H. Freeman, 1994), 141.

3. John Bell, "Against Measurement," in Sixty-Two Years of Uncertainty: Historical, Philosophical, and Physical Inquiries into the Foundations of Quantum Mechanics, ed. Arthur I. Miller (New York: Plenum, 1990).

4. Quantum mechanics is often interpreted as an incomplete classical theory, for example, where probability, nonlocality, and entanglement are ontologically insignificant epistemic artifacts. These artifacts are consequential of our inability to account for a continuum of classically deterministic "hidden variables," which would otherwise complete the theory. Max Born, in this spirit, demonstrated how quantum mechanical probability outcomes can be equated with classical statistical probability outcomes. This purely classical, statistical interpretation of quantum mechanics has, with respect to nonlocality, since been theoretically disconfirmed by John Bell (J. S. Bell, "On the Einstein Podolsky Rosen Paradox," Physics 1, no. 3 [1964]: 195-200), and later experimentally disconfirmed (A. Aspect, J. Dalibard, and G. Roger, Phys. Rev. Lett 44 [1982]: 1804-1807). Various "mostly classical" nonlocal hidden-variables interpretations, such as the one of David Bohm, became newly championed in response to these disconfirmations. Thus the relegation of quantum mechanics to mere epistemic significance by Bohm's interpretation, in defense of classicality, now 
depends upon the concept of an etherlike field of point-particles causally influencing each other nonlocally, in flagrant violation of the otherwise classical ontology the interpretation was designed to preserve.

5. Niels Bohr, "Discussion with Einstein on Epistemological Problems in Atomic Physics," in Albert Einstein: Philosopher-Scientist, ed. Paul Arthur Schilpp (New York: Harper, 1959), 210.

6. Werner Heisenberg, Physics and Philosophy (New York: Harper Torchbooks, 1958), 54-55.

7. K. Popper, Quantum Theory and the Schism in Physics (New Jersey: Rowman and Littlefield, 1956), 100.

8. Bohr, "Discussion with Einstein," 210.

9. Niels Bohr, Atomic Theory and the Description of Nature (Cambridge: Cambridge University Press, 1934), 1.

10. Niels Bohr, Atomic Physics and Human Knowledge (New York: Wiley, 1958), 18.

11. Bohr, Atomic Theory and the Description of Nature, 1.

12. To posit an ontological characterization of the world as fundamentally unknowable is, of course, to doom the ontology to incoherence by its own reasoning, reducing it to the paradox of Epimenides. An admonition from Whitehead comes to mind: "The requirement of coherence is the great preservative of rationalistic sanity. But the validity of its criticism is not always admitted. If we consider philosophical controversies, we shall find that disputants tend to require coherence from their adversaries, and to grant dispensations to themselves" (Process and Reality, 6).

13. Henry P. Stapp, Mind, Matter, and Quantum Mechanics (Berlin: Springer-Verlag, 1993), 60.

14. William James, Pragmatism and the Meaning of Truth (Cambridge, Mass.: Harvard University Press, 1978), 239.

15. John von Neumann, Mathematical Foundations of Quantum Mechanics (Princeton, N.J.: Princeton University Press, 1955).

16. Ibid., 418-420.

17. Ibid., 352 .

18. Heisenberg, Physics and Philosophy, 41.

19. Werner Heisenberg, "The Development of the Interpretation of the Quantum Theory," in Niels Bohr and the Development of Physics, ed. Wolfgang Pauli (New York: McGraw-Hill, 1955), 12.

20. Erwin Schrödinger, "Die gegenwartige Situation in der Quantenmechanik," Naturwissenschaftern 23 (1935): 807-812, 823-828, 844-849. English translation, John D. Trimmer, Proceedings of the American Philosophical Society 124 (1980): 323-338.

21. E. P. Wigner, in The Scientist Speculates: An Anthology of PartlyBaked Ideas, ed. Irving John Good (New York: Basic Books, 1962), 284. 
22. Walter Heitler, "The Departure from Classical Thought in Modern Physics," in Albert Einstein: Philosopher-Scientist, ed. Paul Arthur Schilpp (New York: Harper, 1959), 194.

23. Heisenberg, Physics and Philosophy, 55.

24. Ibid.

25. Patrick Heelan, S.J., Quantum Mechanics and Objectivity (The Hague: Martinus Nijhoff, 1965), 151.

26. G. Ghirardi, A. Rimini, and P. Pearle, in Sixty-Two Years of Uncertainty, ed. A. Miller (New York: Plenum, 1990), 167, 193.

27. John Bell, Speakable and Unspeakable in Quantum Mechanics (Cambridge: Cambridge University Press, 1987).

28. G. Ghirardi, in Structures and Norms in Science, ed. M. L. Dalla Chiara et al. (Dordrecht: Kluwer Academic, 1997).

29. G. Ghirardi, Physics Today (Letters) April 1993: 15.

30. Hugh Everett III, Rev. Mod. Phys. 29 (1957): 44. See also B. S. DeWitt and N. Graham, eds., The Many Worlds Interpretation of Quantum Mechanics (Princeton, N.J.: Princeton University Press, 1973).

31. Bell, Speakable and Unspeakable in Quantum Mechanics, 159-168.

32. Heisenberg, Physics and Philosophy, 185. 